\title{
Is high-intensity interval cycling feasible and more beneficial than continuous cycling for knee osteoarthritic patients? - Results of a randomised control feasibility trial.
}

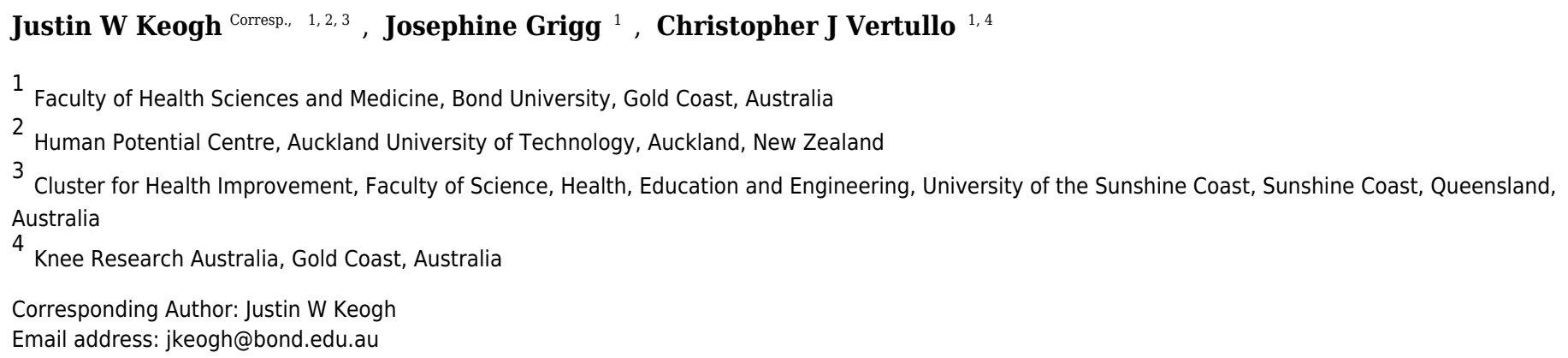

Background. Knee osteoarthritis (OA) patients often suffer joint pain and stiffness, which contributes to negative changes in body composition, strength, physical performance (function), physical activity and health-related quality of life. To reduce these symptoms and side effects of knee OA, moderate intensity continuous cycling (MICT) is often recommended. While resistance training is considered the optimal form of training to improve sarcopenic outcomes, it imposes higher joint loads and requires supervision, either initially or continuously by trained exercise professionals. Therefore, this pilot study sought to gain some insight into the feasibility and potential benefits of high intensity interval training (HIIT) cycling as an alternative exercise option to MICT cycling for individuals with knee OA. Methods. Twenty-seven middle-aged and older adults with knee OA were randomly allocated to either MICT or HIIT, with both programs involving 4 unsupervised home-based cycling sessions ( $\sim 25$ minutes per session) each week for 8 weeks. Feasibility was assessed by enrolment rate, withdrawal rate, exercise adherence and number of adverse effects. Efficacy was assessed by health-related quality of life (WOMAC and Lequesne index), physical function (Timed Up-and-go (TUG), Sit-to-stand (STS) and preferred gait speed) and body composition (body mass, BMI, body fat percentage and muscle mass). Results. Twenty-seven of the interested 50 potential participants (54\%) enrolled in the study, with 17 of the 27 participants completing the trial (withdrawal rate of 37\%); with the primary withdrawal reasons being unrelated injuries or illness or family related issues. Of the 17 participants who completed the trial, exercise adherence was very high (HIIT 94\%; MICT 88\%). While only three individuals (one in the MICT and two in the HIIT group) reported adverse events, a total of 28 adverse events were reported, with 24 of these attributed to one HIIT participant. Pre-post test analyses 
indicated both groups significantly improved their WOMAC scores, with the HIIT group also significantly improving in the TUG and STS. The only significant between-group difference was observed in the TUG, whereby the HIIT group improved significantly more than the MICT group. No significant changes were observed in the Lequesne index, gait speed or body composition for either group. Discussion. An unsupervised home-based HIIT cycle program appears somewhat feasible for middle-aged and older adults with knee OA and may produce similar improvements in health-related quality of life but greater improvements in physical function than MICT. These results need to be confirmed in larger randomised controlled trials to better elucidate the potential for HIIT to improve outcomes for those with knee OA. Additional research needs to identify and modify the potential barriers affecting the initiation and adherence to home-based HIIT cycling exercise programs by individuals with knee OA. 
1 Is high-intensity interval cycling feasible and more beneficial than continuous cycling for knee

2 osteoarthritic patients? - Results of a randomised control feasibility trial.

3 Justin W L Keogh ${ }^{1,2,3}$, Josephine Grigg ${ }^{1}$ and Christopher J Vertullo ${ }^{1,4}$

4

$5 \quad{ }^{1}$ Faculty of Health Science and Medicine, Bond University, Gold Coast, Australia;

$6{ }^{2}$ Human Potential Centre, AUT University, Auckland, New Zealand;

$7 \quad{ }^{3}$ Cluster for Health Improvement, Faculty of Science, Health, Education and Engineering, University of

8 the Sunshine Coast

$9 \quad{ }^{4}$ Knee Research Australia, Gold Coast, Australia

10

11 Corresponding author

12 Justin Keogh

13 Faculty of Health Sciences and Medicine

14 Bond University

15 University Drive, Robina

16 Queensland, 4229

17 Australia

18 jkeogh@bond.edu.au

$19 \quad 61755954487$ 
21 Is high-intensity interval cycling feasible and more beneficial than continuous cycling for knee

22 osteoarthritic patients? - Results of a randomised control feasibility trial.

\section{Abstract}

Background. Knee osteoarthritis (OA) patients often suffer joint pain and stiffness, which contributes to negative changes in body composition, strength, physical performance (function), physical activity and health-related quality of life. To reduce these symptoms and side effects of knee OA, moderate intensity continuous cycling (MICT) is often recommended. While resistance training is considered the optimal form of training to improve sarcopenic outcomes, it imposes higher joint loads and requires supervision, either initially or continuously by trained exercise professionals. Therefore, this pilot study sought to gain some insight into the feasibility and potential benefits of high intensity interval training (HIIT) cycling as an alternative exercise option to MICT cycling for individuals with knee OA.

Methods. Twenty-seven middle-aged and older adults with knee OA were randomly allocated to either MICT or HIIT, with both programs involving 4 unsupervised home-based cycling sessions ( 25 minutes per session) each week for 8 weeks. Feasibility was assessed by enrolment rate, withdrawal rate, exercise adherence and number of adverse effects. Efficacy was assessed by health-related quality of life (WOMAC and Lequesne index), physical function (Timed Up-and-go (TUG), Sit-to-stand (STS) and preferred gait speed) and body composition (body mass, BMI, body fat percentage and muscle mass).

Results. Twenty-seven of the interested 50 potential participants (54\%) enrolled in the study, with 17 of the 27 participants completing the trial (withdrawal rate of 37\%); with the primary withdrawal reasons being unrelated injuries or illness or family related issues. Of the 17 participants who completed the trial, exercise adherence was very high (HIIT 94\%; MICT 88\%). While only three individuals (one in the MICT and two in the HIIT group) reported adverse events, a total of 28 adverse events were reported, with 24 of these attributed to one HIIT participant. Pre-post test analyses indicated both groups 
44 significantly improved their WOMAC scores, with the HIIT group also significantly improving in the TUG

45 and STS. The only significant between-group difference was observed in the TUG, whereby the HIIT

46 group improved significantly more than the MICT group. No significant changes were observed in the

47 Lequesne index, gait speed or body composition for either group.

48 Discussion. An unsupervised home-based HIIT cycle program appears somewhat feasible for middle-

49 aged and older adults with knee OA and may produce similar improvements in health-related quality of

50 life but greater improvements in physical function than MICT. These results need to be confirmed in

51 larger randomised controlled trials to better elucidate the potential for HIIT to improve outcomes for

52 those with knee OA. Additional research needs to identify and modify the potential barriers affecting the

53 initiation and adherence to home-based HIIT cycling exercise programs by individuals with knee OA. 


\section{Introduction}

Osteoarthritis $(\mathrm{OA})$ is a highly prevalent degenerative joint disease affecting many middle-aged and older adults, with recent global data indicating OA of the hip and knee were ranked as the 11th highest contributor to global disability and 38th highest in disability adjusted life years (DALYs) of the 291 health conditions analysed (Cross et al. 2014). The knee is one of the most affected osteoarthritic joints, resulting in a range of symptoms including pain and tenderness that typically limit the individuals' physical function and mobility (Flores \& Hochberg 2003; Hootman et al. 2003). Such changes typically result in considerable physical inactivity, with a recent accelerometer cross-sectional study involving 1,111 participants reporting that only $13 \%$ of men and $8 \%$ of women with OA were meeting the recommended levels of aerobic physical activity and that an additional $40 \%$ of men and $57 \%$ of women could be classified as inactive, participating in no bouts of moderate to vigourous physical activity for more than 10 minutes at a time (Dunlop et al. 2011). These knee OA-related reductions in physical function and physical activity may accelerate the age-related loss of muscle mass, muscle strength and function (especially around the knee joint), referred to as sarcopenia (Cruz-Jentoft et al. 2010; Senior et al. 2015); and contribute to an increased risk of obesity and poor cardiovascular health (Roubenoff 2000). Collectively, the symptoms and adverse events associated with the development of knee OA may further compromise health and well-being and contribute to a downward spiral into greater disability, poor health-related quality of life and further health complications (Yoshimura et al. 2012).

Several recent meta-analyses indicate that a variety of forms of exercise significantly improve pain, function and health-related quality of life in individuals with knee OA (Fransen et al. 2015; Tanaka et al. 2015; Uthman et al. 2013). While a number of different modes of exercise have been examined in the studies included in these meta analyses, cycling and aquatic exercise are often the most commonly recommended (Arthritis Foundation) and performed (Hootman et al. 2003) exercise options for 
individuals with knee OA in the community. Such popularity may reflect the benefits and tolerability of these forms of exercise, with aquatic exercise and cycling imposing lower knee joint loads compared to walking, stair climbing and common resistance training exercises such as the leg extension and squat (Hall et al. 2013; Heino Brechter \& Powers 2002; Kutzner et al. 2010; Kutzner et al. 2012; Powers et al. 2014).

The benefits of cycling for middle-aged and older adults with knee OA are also well documented. These include significant improvements in health-related quality of life, as assessed by the WOMAC questionnaire (Alkatan et al. 2016; Salacinski et al. 2012); physical function, as assessed by gait speed (Alkatan et al. 2016; Salacinski et al. 2012) and sit to stand performance (Mangione et al. 1999) and body composition, as assessed by body mass, waist and hip circumference and visceral adipose tissue (Alkatan et al. 2016). As the majority of these cycling studies utilised moderate intensity continuous training (MICT) protocols, exercise prescriptions involving higher intensity muscle contractions may better improve sarcopenic outcomes including muscle mass, strength and function (Landi et al. 2014).

We propose that the utilisation of high intensity interval training (HIIT) may be an exercise approach that simultaneously improves sarcopenic and cardiovascular outcomes in the OA patients, while at the same time having a similar degree of feasibility and tolerability as MICT. High-intensity interval training typically requires participants to alternate short periods ( 8-60 s) of high-intensity activity with longer ( 20-90 s) recovery periods of lower intensity activity (Boutcher et al. 2013; Maclnnis \& Gibala 2017; Shiraev \& Barclay 2012). Based on data obtained from other clinical populations, HIIT cycling may provide a better stimulus for overcoming the knee OA related sarcopenic outcomes (i.e. loss in leg muscle mass, strength and function (Alvarez et al. 2017; Jia et al. 2018), while still providing a range of cardiometabolic benefits than is currently achieved by MICT exercise mode such as cycling or aquatic exercise (Francois \& Little 2015; Liou et al. 2016; Ramos et al. 2015; Shiraev \& Barclay 2012). 
101 Unfortunately, almost no research has assessed the feasibility and potential benefits of HIIT in

102 musculoskeletal conditions like knee OA. This may reflect the concern that some health professionals

103 may have regarding the potential musculoskeletal and cardiovascular health risks associated with HIIT.

104 While HIIT appears well tolerated by older and middle-aged individuals with a variety of cardiovascular

105 diseases, even when performed at home (Aamot et al. 2014; Rognmo et al. 2012), only one study

106 appears to have assessed the safety of HIIT in arthritic populations. This recent pilot study involving

107 young to middle-aged adults with rheumatoid arthritis $(n=7)$ and juvenile idiopathic arthritis $(n=11)$,

108 reported no significant change in disease activity or pain during the 10 week HIIT and MICT cycling

109 exercise program (Sandstad et al. 2015).

110 Therefore, this randomised control pilot trial was conducted to compare two unsupervised home-based

111 (HIIT and MICT) cycling exercise programs in terms of their relative feasibility and ability to improve

112 health-related quality of life, physical function and body composition in middle-aged and older knee OA

113 patients. It was hypothesised that both forms of home-based cycling would be feasible and produce a

114 range of benefits for the participants.

115

116 Methods

117 Research design

118 A study protocol for this trial has been previously published (Keogh et al. 2017), with a summary of the

119 key details provided below. According to a recent conceptual framework paper for feasibility and pilot

120 studies (Eldridge et al. 2016), the current study can be described as a "randomised pilot study". A variety

121 of approaches were used to recruit participants including discussions with physicians and

122 physiotherapists as well as media stories published in selected local magazines and newsletters that had 
123 some relevance to middle-aged and older adults. As this was a randomised pilot study that focused on

124 the feasibility of a novel exercise program that hasn't been investigated in middle-aged and older adults 125 with knee OA, no specific power analysis was performed.

126 Prior to performing the baseline assessments, all potential participants were screened for suitability to

127 participate in the exercise program by their physician; with this supplemented by the Australian

128 Association of Exercise and Sports Science (ESSA) pre-screening exercise form. Once the baseline

129 assessments were completed, participants were randomised to the appropriate intervention and

130 provided with a written explanation of how to perform their exercise program, either at home or in a

131 gymnasium. The participants were also given a practical familiarisation on a Monark cycle ergometer on

132 how to perform their exercise program and how to set up their bike appropriately to minimise the risk

133 of additional knee pain. While there was no formal contact organised with the research team during the

134 intervention period, participants were told to contact the team should any problems arise with the

135 program, or if they had any questions.

136 The randomisation to either of the two cycling groups (HIIT or MICT) involved a computer-generated

137 randomisation sequence (https://www.random.org/) performed by the lead investigator who had no

138 interaction with the participants in relation to their assessments. This randomisation sequence was

139 concealed in an Excel spreadsheet until it was retrieved by the research assistant immediately after

140 completing the baseline assessment of each participant. As a result, the research assistant who

141 informed the participants of their group allocation and conducted all the assessments was unable to be

142 blinded to participant allocation. The participants were unable to be blinded to their participation in the 143 exercise trial.

144 The protocol for this study was approved by the Bond University Research Ethics Committee (RO1776)

145 and registered at the ANZCTR (trial registration number ACTRN12616000273482). All eligible 
146 participants provided written consent before participating in this project. The trial recruited the first

147 participant in May 2014, with data collection completed by January 2016 as the research assistant would

148 not be available after this time.

149 Participants

150 Males and females between the age of 40 and 80 years with a confirmed diagnosis of knee osteoarthritis

151 by an orthopaedic surgeon were eligible to participate in this study. Their physician also had to provide

152 clearance for them to participate in the study and to state that the patient was unlikely to change their

153 osteoarthritic management during the study. As the cycling programs were home-based, all participants

154 also needed to have access to a stationary bicycle to be eligible to participate. Participants were allowed

155 other comorbidities, if these comorbidities did not contraindicate home-based, unsupervised exercise.

156 The conditions which were contraindications to participation in this study included unstable cardiac

157 conditions, including a history of arrhythmia and cardiac ischaemia.

Exercise program

159

160

161

162

163

164

All participants were requested to perform 4 stationary cycling sessions per week for 8 weeks, with each session 25 minutes long. The MICT group commenced each session with a 3 minute warm-up at a light intensity and finished with a 2 minute cooldown at a similar intensity to the warm-up. The MICT component required the participants to select a workload that they could cycle at a cadence of $60-80$ rpm for 20 minutes at a moderate intensity, which was defined as "an intensity in which you are able to speak in complete sentences during the exercise. If you find yourself getting too puffed or out of breath - slow down a little". For HIIT participants, their training sessions commenced with a 7 minute warm-up of progressively increasing intensity and concluded with a 6 to 7 minute cool-down of light to moderate cycling. The HIIT component of the training session involved five series of high and low intensity cycling. For the five, $45 \mathrm{~s}$ high-intensity bouts, participants were requested to cycle at a higher cadence (up to 
$169110 \mathrm{rpm}$ ) for $45 \mathrm{~s}$ using a resistance similar or slightly higher than the low intensity recovery bouts,

170 which were to be performed at $70 \mathrm{rpm}$ for a duration of $90 \mathrm{~s}$. The intensity of the high-intensity bouts

171 was defined as "an intensity at which you felt it was quite difficult to complete sentences during the

172 exercise".

173 To minimise the potential for any adverse events relating to the initiation of the exercise program, the

174 participants were encouraged to be somewhat conservative with respect to the intensity of their initial

175 sessions by decreasing the recommended cadence or workload described in the previous paragraph. If

176 they tolerated their first few exercise sessions with no cardiovascular or joint pain, they were requested

177 to progressively increase exercise intensity to that described in the previous paragraph.

Data collection

Outcome measures were collected at baseline and at the end of the eight week cycling programs at a university clinic that the participants were required to visit. To gain some insight into the relative feasibility and safety of the HIIT compared to the MICT cycling program, participants were given a training and adverse events diary. The participants were requested to record the date of each training sessions they performed across each of the eight training weeks. Similarly, the adverse events diary required the participants to state the number of any adverse effects they experienced (if any) and to provide some description of what happened.

Consistent with recent feasibility studies of under-researched exercise programs for a range of older 
191 calculated by dividing the number of individuals who consented to participate in the study by the total

192 number of individuals who expressed interest in participating. The withdrawal rate (also expressed as a 193 percentage) was calculated by dividing the number of participants who failed to complete post-testing

194 by the number of participants who completed baseline testing. Adherence rate was calculated by

195 dividing the number of training sessions completed by each participant by the requested number of

196 training sessions ( $n=32$ ), with this presented as a percentage. Adverse events were defined as "an

197 exercise-induced change that worsens an aspect of your condition that is greater than expected day-to-

198 day variation", a definition very similar to that used previously in the study of 1687 men and women

199 undergoing exercise programs (Bouchard et al. 2012).

200 Efficacy outcomes

201 Efficacy outcomes included two OA-specific health-related quality of life questionnaires (Western

202 Ontario and McMaster Universities Osteoarthritis Index (WOMAC) and the Lequesne Index), three

203 objective physical performance tests (30 s sit to stand (STS), Timed Up and Go (TUG) and habitual gait

204 speed tests) and a body composition assessment (via the Tanita MC-980MA body composition analyser).

205 A brief description of these efficacy outcomes is provided below.

206 WOMAC: The WOMAC is a valid and reliable health-related quality of life questionnaire consisting of 24

207 items that assesses the OA patient's degree of pain (five items), stiffness (two items) and physical

208 function/disability (17 items) typically felt when performing a range of activities of daily living (Bellamy

209 1989; Bellamy et al. 1988). The maximum score for the WOMAC was 96, with a score of 39 or greater

210 indicative of severe arthritis (Hawker et al. 2000).

211 Lequesne index of severity for osteoarthritis of the knee: The Lequesne index is another valid and

212 reliable knee OA health-related quality of life questionnaire that consists of 11 items that assesses the

213 patient's pain/discomfort (5 items), maximum walking distance with or without walking aids (2 items) 
214 and physical function/disability (4 items) (Lequesne 1991; Lequesne 1997). The maximum possible score

215 is 24 , with the severity of the knee OA-related disability described as small (1-4), intermediate (5-7),

216 serious (8-10), very serious (11-13) and extremely serious ( $\geq 14)$ (Lequesne 1991; Lequesne 1997).

217 STS: The STS test is a valid and reliable measure of lower extremity strength and function in older

218 populations including those with knee OA (Lord et al. 2002). Participants were asked to sit on a straight-

219 backed, armless chair that was $\sim 43 \mathrm{~cm}$ in height and keep their arms crossed across the chest. On the

220 word "Go", participants completed as meeting sit to stands as possible in $30 \mathrm{~s}$. Participants performed

221 one practice trial of 3-4 repetitions at a submaximal intensity prior to performing the one trial of this

222 exercise.

223 TUG: The TUG is a valid and reliable measure of functional mobility for a range of older adult

224 populations, including those with knee OA (Podsiadlo \& Richardson 1991). Participants were required to

225 stand up from a $\sim 43 \mathrm{~cm}$ high, armless chair and then proceed to walk around a cone $3 \mathrm{~m}$ away before

226 sitting back on the chair (Podsiadlo \& Richardson 1991), with the following instructions "Stand-up and

227 walk around the flagpole and sit back down on the chair at a pace comfortable for you". Participants

228 were allowed one practice trial, with the best time from three timed trials used for analysis.

229 Habitual gait speed: Habitual gait speed was quantified using the GaitMat II pressure mat system

230 (Manufacturer is EQInc; Model is GaitMat II) (Rosano et al. 2008; Trehan et al. 2015). All gait speed trials

231 were initiated $2 \mathrm{~m}$ (6.56 ft.) before the GaitMat II platform ( $3.66 \mathrm{~m}$ long) and finished $2 \mathrm{~m}$ after the

232 GaitMat II to reduce the potential acceleration and deceleration effects on mean gait speed (Kressig \&

233 Beauchet 2006). Participants were provided with the following instructions "Walk towards the end of

234 the room at a pace that is comfortable for you" (Fien et al. 2016). The average gait speed from three

235 attempts was used for data analysis. 
236 Body composition: Body composition (proportion of muscle, fat and bone) was assessed using the

237 Tanita MC-980MA body composition analyser (Tokyo, Japan) (Ragini B. et al. 2015). As the bio-electrical

238 impedance assessment (BIA) method is sensitive to alterations in hydration, all participants were

239 requested to be normally hydrated and to have not eaten or exercised for a period of two hours before

240 the BIA assessment. All participants stood in a stationary position in bare feet on the Tanita MC-980MA

241 platform while holding the handles for a period of $30 \mathrm{~s}$. According to the manufacturer's user manual,

242 the sensitivity of this device was $0.1 \mathrm{~kg}$ for total body mass, muscle mass, fat-free mass and fat mass.

\section{Statistical analysis}

244 Centrality and dispersion of the continuous data was reported as means and standard deviations,

whereas categorical measures were reported as number and percentage. To test for whether there

were significant differences at baseline between the two groups, two tailed independent t-tests were

performed for continuous variables and chi-square analysis for categorical variables. The chi-square

analysis was performed using an online calculator

http://www.socscistatistics.com/tests/chisquare2/Default2.aspx Two tailed paired t-tests were used to determine within-group changes i.e. pre-post test changes for each of the groups independently. The

https://effect-size-calculator.herokuapp.com/ Effect sizes were described as being small ( $d=0.20-0.49)$, 


\section{Results}

261 The demographic characteristics of the participants who completed the HIIT and MCIT cycle programs

262 are described in Table 1. There were no significant differences between the groups for any of the

263 outcome measures at baseline $(p=0.09-0.94)$.

264

265

Insert Table 1 about here

266

267

A summary of the key feasibility outcomes are presented in Figure 1 and Table 2 . As seen in Figure 1, a total of 27 individuals from the 50 (54\% enrolment rate) who were invited/expressed interest to participate were enrolled in this study. As only 17 of the 27 participants completed the trial, the trial had a withdrawal rate of $\sim 37 \%$, with the most common reasons provided for withdrawal reflecting unrelated

271 illness, unrelated injury or family related issues.

Insert Figure 1 about here (88\%) groups. Three participants (one in the MICT group and two in the HIIT group) reported adverse events during the eight-week study, with the one individual in the MICT group reporting two adverse events relating to discomfort and pain they felt was caused by the bicycle seat. In contrast, 26 adverse 
279

280

281

282

283

284

285

286

287

Insert Table 3 about here

Insert Table 2 about here

events were reported for the HIIT group. Of these 26 adverse events, 24 were reported by one individual who stated that the cycling program aggravated their Bakers cyst behind their knee. Interestingly, this individual still completed 25 of the requested 32 exercise sessions.

The potential efficacy of the two home-based cycling programs with respect to improving the participants' health-related quality of life, physical performance (function) and body composition is summarised in Table 3. The HIIT group demonstrated significant pre-post test improvements in WOMAC, TUG and sit to stand performance over the course of the training program; with the MICT demonstrating a significant pre-post test improvement in the WOMAC only. Based on effect size calculations, all of these significant improvements could be described as moderate effects, with the exception of the WOMAC change for the HIIT group which would be considered a large effect. When comparing the changes between the two groups, the only significant between-group difference was observed for the TUG, whereby the HIIT group improved to a significantly greater extent than the MICT group. No significant improvements were observed in the Lequesne index or any of the body composition outcomes for either of the two groups.

\section{Discussion}


299 The primary aim of this pilot study was to examine and compare the feasibility and safety of

300 unsupervised, home-based HIIT and MICT cycling in middle-aged and older adults with knee OA; with

301 the secondary aim being to gain some insight into the relative efficacy of these forms of cycling for

302 improving health-related quality of life, physical performance and body composition.

303 The results of the current study are heterogeneous with regards to the feasibility of the two home-

304 based cycling programs for middle-aged and older individuals with knee OA. For example, the research

305 team took 1.5 years to identify the 50 potential middle-aged and older individuals with knee OA. The

306 relatively slow recruitment to the study may reflect a variety of factors, perhaps including concerns from

307 potential participants, medical practitioners and physiotherapists that the HIIT exercise program may

308 aggravate knee OA symptoms or perhaps that relatively few individuals with knee OA had access to a

309 suitable stationary bicycle. Qualitative and/or quantitative studies examining these potential barriers, as

310 well as the facilitators and motives to home-based HIIT cycling may need to be conducted as has been

311 done recently for individuals with knee OA performing group-based aquatic exercise programs (Fisken et

312 al. 2015; Fisken et al. 2014), to further improve these recruitment rates.

313 It must also be acknowledged that only $54 \%$ of the potentially interested 50 individuals enrolled in this

314 exercise study. While this enrolment rate is relatively low it should be noted that a total of 15 potential

315 participants ( $30 \%$ of those were initially interested) were deemed ineligible, with the majority of these

316 individuals being located too far away to come in for baseline and post tests or due to a lack of access to

317 a stationary bike. Furthermore, the $54 \%$ enrolment rate of the current study was slightly higher than the

31844 and 46\%, respectively reported by Mangione et al. (1999) and Rewald et al. (2015), who also

319 performed cycling interventions for middle-aged and older adults with knee OA. Such relatively low

320 enrolment rates further support the need for more research needs to investigate the barriers to

321 enrolment in such cycling studies. 
322 Our results also indicated that only 17 of the 27 (63\%) of the original participants completed the cycling

323 program, with most of the reasons for withdrawal not related to the exercise program. This withdrawal

324 rate was similar to Salacinski et al. (2012) who reported 68\% of their original participants completed the

325 project; but substantially lower than other cycling studies that observed 83-95\% completion rates

326 (Alkatan et al. 2016; Mangione et al. 1999; Rewald et al. 2015). For those participants who continued in

327 our two cycling programs, a very high level of exercise adherence was reported for both the MICT (88\%)

328 and HIIT (92\%) groups. Such values appear to be consistent (Alkatan et al. 2016; Mangione et al. 1999;

329 Salacinski et al. 2012) or greater (Rewald et al. 2015) than reported in previous cycling studies involving

330 individuals with knee OA.

331 Analysis of the adverse event data indicated that only three participants (one in the MICT and two in the

332 HIIT group) reported any adverse events, defined as "an exercise induced change that worsens an aspect

333 of your condition that is greater than expected day-to-day variation" over the course of the intervention.

334 Two of these individuals each reported two adverse events, with one individual in the HIIT group

335 unfortunately reporting 24 adverse events over the course of the 25 exercise sessions they performed.

336 While the proportion of participants reporting adverse events (HIIT; 2 out of 9, 22\%; MICT, 1 out of 8 ,

337 13\%) and a total of 24 adverse events for just one participant in the HIIT group appears very high, it

338 needs to be remembered that this is an unsupervised exercise trial involving middle-aged and older

339 adults with knee osteoarthritis. Further, it could be argued that the severity of these adverse events was

340 quite low as the even the individual who reported 24 further adverse events continued to perform the

341 majority of their requested exercise sessions even though such cycling aggravated their Bakers cyst.

342 While such adverse events may therefore not truly reflect the individual's knee OA diagnosis, some

343 caution may need to be made when prescribing cycling, especially of a HIIT nature to individuals with

344 Bakers cysts; and to perhaps include Bakers cysts as an exclusion criteria for future cycling studies. 
345 Comparison of our adverse data to that of literature was also difficult as some cycling studies did not

346 report such data (Alkatan et al. 2016; Mangione et al. 1999) and even those studies that did, no clear

347 definition of adverse events was often given (Rewald et al. 2015; Salacinski et al. 2012). It was also

348 observed that some studies stated that no adverse events occurred but then indicated that a number of

349 individuals withdrew from the study due to knee pain (Alkatan et al. 2016; Salacinski et al. 2012),

350 although it was not explicitly stated whether such knee pain was considered an adverse event or

351 whether it was related to the exercise program.

352 According to a recent review by Wellsandt and Golightly (2018), adherence and the level of

353 improvement resulting from an exercise program for individuals with knee OA may be influenced by a

354 variety of factors including the participant's preference for the degree of supervision and mode of

355 exercise. This suggests that the true feasibility of the home-based HIIT and MICT cycling observed in the

356 present study could be higher in individuals with knee OA who are able to select their preferred exercise

357 activity. Such a finding would suggest that clinicians and exercise professionals should endeavour to find

358 out the exercise preferences of their patients and match these where possible to the efficacy evidence

359 reported in RCTs and meta analyses.

360 Regarding the efficacy outcomes, both cycling programs demonstrated some significant benefits for the

361 participants. Both groups significantly improved their health-related quality of life as measured by the

362 WOMAC, but not the Lequesne index. The significant improvements in their WOMAC score (HIIT: 14.9

363 points; MICT: 11.9 points) were comparable or greater than the 10.6-11.6 point improvements reported

364 in the literature for other cycling exercise studies involving knee OA participants (Alkatan et al. 2016;

365 Salacinski et al. 2012). The significant increases in the WOMAC score in the current study appear

366 clinically significant as they exceed the 9 point improvement identified by Tubach et al. (2005) as being a

367 clinically relevant change. While the improvements in the Lequesne index were non-significant (HIIT: $p=$ 
0.102; MICT: $p=0.081$ ), both groups reported small effect size improvements the HIIT group had a reduction of $\sim 2$ points on the scale that resulted in their overall group being classified as "intermediate disability" at post test compared to their baseline classification of "serious disability" (Lequesne 1991;

371 Lequesne 1997).

372 The tendencies for the participants to report improved health-related quality of life, including reduced pain, stiffness and disability were consistent with some of the functional changes reported for the HIIT group. Specifically, the HIIT group significantly improved their TUG and STS performance, with the change in TUG performance significantly greater than the MICT group. The magnitude of these changes for the HIIT group (TUG: -1.1 s; STS: +2 repetitions) would appear clinically significant based on the minimally detectable change of $1.1 \mathrm{~s}$ for the TUG (Alghadir et al. 2015) and 1.64 repetitions for the STS

378 (Gill \& McBurney 2008) reported in previous studies for individuals with knee and/or hip OA. It must however be acknowledged that neither exercise group significantly improved their habitual gait speed over the $3.66 \mathrm{~m}$ course. Such a lack of change in gait speed was consistent with the low $(40 \%$ of heart rate reserve) and high (70\% of heart rate reserve) intensity MICT cycle training groups in the study of Mangione et al. (1999), but inconsistent with two cycling studies for individuals with knee OA who reported increases of between $0.08-0.20 \mathrm{~m} / \mathrm{s}$ over distances of 3.66 to $6 \mathrm{~m}$ (Alkatan et al. 2016;

Salacinski et al. 2012).

The lack of any significant changes in body composition for either of the two groups in the current study appears relatively inconsistent with the very limited number of cycling studies assessing these outcomes for individuals with knee OA. Specifically, Alkatan et al. (2016) reported that the cycling group significantly improved body mass $(-1.5 \mathrm{~kg})$, waist circumference $(-3 \mathrm{~cm})$, hip circumference $(-2 \mathrm{~cm})$ and visceral adipose tissue (-0.1 kg); although no significant changes were observed for BMI, body fat 
391 al. (2016) may reflect two primary factors. The first is that since Alkatan et al. (2016) used DEXA

392 compared to our study's BIA, there is the potential that the increased sensitivity of the DEXA was

393 required to observe such body composition changes over relatively small training periods. Further, the

394 duration of the cycling program for Alkatan et al. (2016) was 1.5 times longer (12 vs eight weeks) than

395 the current study. The potential for cycling, particularly the novel HIIT cycling program examined in this

396 study to improve body composition may also be influenced by the exercise preferences of the

397 individuals with knee OA. In a recent review, Wellsandt and Golightly (2018) found that exercise

398 preference (e.g. degree of supervision and exercise mode) contributes to exercise adherence and to the

399 magnitude of change in body composition outcomes with exercise in individuals with OA. Specifically,

400 studies which randomly allocated participants to an exercise program reported no significant change in

401 body composition, whereas the one study that allowed participants to choose their exercise program

402 (Loew et al. 2017), reported significant reductions in body mass; with those adhering to the walking

403 program also reporting significantly greater reductions in waist circumference.

404 There were however several limitations associated with the current study. The first limitation reflects

405 the self-report nature of much of the data including the WOMAC and Lequesne index as well as the

406 training and adverse events diary. Even though the participants typically completed their training and

407 adverse event diaries, the actual exercise dose performed is always difficult to quantify during home-

408 based exercise, as no measure of external (power output) or internal (heart rate) workload was

409 collected from the participants. It is also acknowledged that the home-based nature of the intervention

410 may not have optimised the potential benefits of the HIIT program as a trained exercise professional

411 supervising the exercise sessions would typically monitor and progressively increase workloads over the

412 course of the training program. The sample size was also relatively small, which may affect our ability to

413 detect within and between group significant differences and the potential generalisability of the trial

414 findings. Nevertheless, the sample size in the study was greater (Rewald et al. 2015) than or somewhat 
415 comparable (Mangione et al. 1999; Salacinski et al. 2012) to some other cycling studies involving middle-

416 aged and older adults with knee OA.

\section{Conclusions}

418 Our results are somewhat supportive of the feasibility of home-based HIIT and MICT cycling programs

419 for middle-aged and older adults with knee OA, with the enrolment rate and adherence rate for both

420 the HIIT and MICT groups comparable to other cycling studies involving similar populations (Alkatan et al.

421 2016; Rewald et al. 2015; Salacinski et al. 2012). The number of adverse events (HIIT: 2 of 9 participants,

42226 adverse events; MICT: 1 of 8 participants, 2 adverse events) appeared very high, although 24 of these

423 adverse events were reported by one HIIT individual with a Bakers cyst on their knee, who still

424 completed 25 of the requested 32 exercise sessions. Efficacy data demonstrated significant benefits in

425 health-related quality of life (WOMAC) for both groups, with the HIIT group also reporting significant

426 increases in functional performance as assessed by TUG and STS. The major limitation of this study is the

427 small sample size, which may limit our statistical power and increase our chance of type Il error. This

428 suggests that larger scale RCTs should further investigate the feasibility and efficacy of HIIT compared to

429 MICT cycling for a variety of knee OA sub-populations; including studies comparing standard supervised

430 and home-based exercise programs. 
432

433

434

435

436

437

438

439

440

441

442

443

444

445

446

447

448

449

450

451

452

453

454

455

456

457

458

459

460

461

462

463

464

465

466

467

468

469

470

471

472

473

474

475

476

477

478

479

\section{References}

Aamot IL, Forbord SH, Gustad K, Lockra V, Stensen A, Berg AT, Dalen H, Karlsen T, Stoylen A. 2014. Home-based versus hospital-based high-intensity interval training in cardiac rehabilitation: a randomized study. European journal of preventive cardiology 21(9):1070-8.

Alghadir A, Anwer S, Brismée J-M. 2015. The reliability and minimal detectable change of Timed Up and Go test in individuals with grade $1-3$ knee osteoarthritis. BMC Musculoskeletal Disorders 16:174.

Alkatan M, Baker JR, Machin DR, Park W, Akkari AS, Pasha EP, Tanaka H. 2016. Improved Function and Reduced Pain after Swimming and Cycling Training in Patients with Osteoarthritis. J Rheumatol 43(3):666-72.

Alvarez C, Ramirez-Campillo R, Ramirez-Velez R, Izquierdo M. 2017. Prevalence of Non-responders for Glucose Control Markers after 10 Weeks of High-Intensity Interval Training in Adult Women with Higher and Lower Insulin Resistance. Front Physiol 8:479.

Arthritis Foundation. Your exercise solution - biking [Internet]. Arthritis Foundation. Available from: https://www.arthritis.org/living-with-arthritis/exercise/arthritis-friendly/biking.php

Bellamy N. 1989. Pain assessment in osteoarthritis: experience with the WOMAC osteoarthritis index. Semin Arthritis Rheum 18(4 Suppl 2):14-7.

Bellamy N, Buchanan WW, Goldsmith CH, Campbell J, Stitt LW. 1988. Validation study of WOMAC: a health status instrument for measuring clinically important patient relevant outcomes to antirheumatic drug therapy in patients with osteoarthritis of the hip or knee. J Rheumatol 15(12):1833-40.

Bossers WJR, Scherder EJA, Boersma F, Hortobágyi T, van der Woude LHV, van Heuvelen MJG. 2014. Feasibility of a Combined Aerobic and Strength Training Program and Its Effects on Cognitive and Physical Function in Institutionalized Dementia Patients. A Pilot Study. PLOS ONE 9(5):e97577.

Bouchard C, Blair SN, Church TS, Earnest CP, Hagberg JM, Häkkinen K, Jenkins NT, Karavirta L, Kraus WE, Leon AS, Rao DC, Sarzynski MA, Skinner JS, Slentz CA, Rankinen T. 2012. Adverse Metabolic Response to Regular Exercise: Is It a Rare or Common Occurrence? PLOS ONE 7(5):e37887.

Boutcher SH, Park Y, Dunn SL, Boutcher YN. 2013. The relationship between cardiac autonomic function and maximal oxygen uptake response to high-intensity intermittent-exercise training. J Sports Sci 31(9):1024-9.

Cheema BS, Davies TB, Stewart M, Papalia S, Atlantis E. 2015. The feasibility and effectiveness of highintensity boxing training versus moderate-intensity brisk walking in adults with abdominal obesity: a pilot study. BMC Sports Sci Med Rehabil 7:3.

Cross $M$, Smith E, Hoy D, Nolte S, Ackerman I, Fransen M, Bridgett L, Williams S, Guillemin F, Hill CL, Laslett LL, Jones G, Cicuttini F, Osborne R, Vos T, Buchbinder R, Woolf A, March L. 2014. The global burden of hip and knee osteoarthritis: estimates from the Global Burden of Disease 2010 study. Annals of the Rheumatic Diseases 73(7):1323-1330.

Cruz-Jentoft AJ, Baeyens JP, Bauer JM, Boirie Y, Cederholm T, Landi F, Martin FC, Michel JP, Rolland Y, Schneider SM, Topinkova E, Vandewoude M, Zamboni M. 2010. Sarcopenia: European consensus on definition and diagnosis: Report of the European Working Group on Sarcopenia in Older People. Age and Ageing 39(4):412-23.

Dunlop DD, Song J, Semanik PA, Chang RW, Sharma L, Bathon JM, Eaton CB, Hochberg MC, Jackson RD, Kwoh CK, Mysiw WJ, Nevitt MC, Hootman JM. 2011. Objective physical activity measurement in the osteoarthritis initiative: Are guidelines being met? Arthritis \& Rheumatism 63(11):3372-3382.

Eldridge SM, Lancaster GA, Campbell MJ, Thabane L, Hopewell S, Coleman CL, Bond CM. 2016. Defining Feasibility and Pilot Studies in Preparation for Randomised Controlled Trials: Development of a Conceptual Framework. PLOS ONE 11(3):e0150205. 
480

481

482

483

484

485

486

487

488

489

490

491

492

493

494

495

496

497

498

499

500

501

502

503

504

505

506

507

508

509

510

511

512

513

514

515

516

517

518

519

520

521

522

523

524

525

526

527

Fien S, Henwood T, Climstein M, Keogh JWL. 2016. Feasibility and benefits of group-based exercise in residential aged care adults: a pilot study for the GrACE programme. PeerJ 4:e2018.

Fisken A, Keogh JWL, Waters D, Hing W. 2015. Perceived benefits, motives, and barriers to aqua-based exercise among older adults with and without osteoarthritis. Journal of Applied Gerontology 34(3):377-396.

Fisken A, Waters D, Hing W, Keogh JWL. 2014. Perception and responses to different forms of aquabased exercise among older adults with osteoarthritis. International Journal of Aquatic Research and Education 8(1):32-52.

Flores R, H. , Hochberg MC. 2003. Definition and classification of osteoarthritis. In: Brandt KD, Dohert, M. and Lohmander, L. S., editor. Osteoarthritis. 2nd ed. Oxford: Oxford University Press.

Francois ME, Little JP. 2015. Effectiveness and safety of high-intensity interval training in patients with type 2 diabetes. Diabetes Spectrum 28(1):39-44.

Fransen M, McConnell S, Harmer AR, Van der Esch M, Simic M, Bennell KL. 2015. Exercise for osteoarthritis of the knee. Cochrane Database Syst Rev 1:Cd004376.

Gill S, McBurney H. 2008. Reliability of performance-based measures in people awaiting joint replacement surgery of the hip or knee. Physiother Res Int 13(3):141-52.

Hall M, Boyer ER, Gillette JC, Mirka GA. 2013. Medial knee joint loading during stair ambulation and walking while carrying loads. Gait Posture 37(3):460-2.

Hawker GA, Wright JG, Coyte PC, Williams JI, Harvey B, Glazier R, Badley EM. 2000. Differences between men and women in the rate of use of hip and knee arthroplasty. New England Journal of Medicine 342(14):1016-1022.

Heino Brechter J, Powers CM. 2002. Patellofemoral stress during walking in persons with and without patellofemoral pain. Med Sci Sports Exerc 34(10):1582-93.

Hootman JM, Macera CA, Ham SA, Helmick CG, Sniezek JE. 2003. Physical activity levels among the general US adult population and in adults with and without arthritis. Arthritis \& Rheumatism 49:129-135.

Jia D, Cai M, Xi Y, Du S, ZhenjunTian. 2018. Interval exercise training increases LIF expression and prevents myocardial infarction-induced skeletal muscle atrophy in rats. Life Sci 193:77-86.

Keogh JW, Grigg J, Vertullo CJ. 2017. Is home-based, high-intensity interval training cycling feasible and safe for patients with knee osteoarthritis: study protocol for a randomised pilot study? Orthopedic Journal of Sports Medicine 5(3):2325967117694334.

Kressig RW, Beauchet O. 2006. Guidelines for clinical applications of spatio-temporal gait analysis in older adults. Aging Clin Exp Res 18(2):174-176.

Kutzner I, Heinlein B, Graichen F, Bender A, Rohlmann A, Halder A, Beier A, Bergmann G. 2010. Loading of the knee joint during activities of daily living measured in vivo in five subjects. J Biomech 43(11):2164-73.

Kutzner I, Heinlein B, Graichen F, RohImann A, Halder AM, Beier A, Bergmann G. 2012. Loading of the knee joint during ergometer cycling: telemetric in vivo data. J Orthop Sports Phys Ther 42(12):1032-8.

Landi F, Marzetti E, Martone AM, Bernabei R, Onder G. 2014. Exercise as a remedy for sarcopenia. Curr Opin Clin Nutr Metab Care 17(1):25-31.

Lequesne M. 1991. Indices of severity and disease activity for osteoarthritis. Semin Arthritis Rheum 20(6 Suppl 2):48-54.

Lequesne MG. 1997. The algofunctional indices for hip and knee osteoarthritis. J Rheumatol 24(4):77981.

Liou K, Ho S, Fildes J, Ooi SY. 2016. High Intensity Interval versus Moderate Intensity Continuous Training in Patients with Coronary Artery Disease: A Meta-analysis of Physiological and Clinical Parameters. Heart, Lung and Circulation 25(2):166-74. 
528

529

530

531

532

533

534

535

536

537

538

539

540

541

542

543

544

545

546

547

548

549

550

551

552

553

554

555

556

557

558

559

560

561

562

563

564

565

566

567

568

569

570

571

572

573

574

575

Loew L, Brosseau L, Kenny GP, Durand-Bush N, Poitras S, De Angelis G, Wells GA. 2017. An evidencebased walking program among older people with knee osteoarthritis: the PEP (participant exercise preference) pilot randomized controlled trial. Clin Rheumatol 36(7):1607-1616.

Lord SR, Murray SM, Chapman K, Munro B, Tiedemann A. 2002. Sit-to-stand performance depends on sensation, speed, balance, and psychological status in addition to strength in older people. Journal of Gerontology: Medical Sciences 57A(8):M539-M543.

MacInnis MJ, Gibala MJ. 2017. Physiological adaptations to interval training and the role of exercise intensity. The Journal of Physiology 595(9):2915-2930.

Mangione KK, McCully K, Gloviak A, Lefebvre I, Hofmann M, Craik R. 1999. The effects of high-intensity and low-intensity cycle ergometry in older adults with knee osteoarthritis. Journal of Gerontology: Medical Sciences 54(4):M184-90.

Podsiadlo D, Richardson S. 1991. The timed "Up \& Go": a test of basic functional mobility for frail elderly persons. Journal of the American Geriatrics Society 39:142-148.

Powers CM, Ho KY, Chen YJ, Souza RB, Farrokhi S. 2014. Patellofemoral joint stress during weightbearing and non-weight-bearing quadriceps exercises. J Orthop Sports Phys Ther 44(5):320-7.

Ragini B., Aishwarya S. R., Tamil Selvan M., Pillai A, M A. 2015. Prediction of body fat using segmental body composition by bioelectrical impedance in the evaluation of obesity J Eng Appl Sci 10(8):3627-3634.

Ramos JS, Dalleck LC, Tjonna AE, Beetham KS, Coombes JS. 2015. The impact of high-intensity interval training versus moderate-intensity continuous training on vascular function: a systematic review and meta-analysis. Sports Med 45(5):679-92.

Rewald S, Mesters I, Emans PJ, Arts JJ, Lenssen AF, de Bie RA. 2015. Aquatic circuit training including aqua-cycling in patients with knee osteoarthritis: A feasibility study. $J$ Rehabil Med 47(4):376-81.

Rognmo O, Moholdt T, Bakken H, Hole T, Molstad P, Myhr NE, Grimsmo J, Wisloff U. 2012. Cardiovascular risk of high- versus moderate-intensity aerobic exercise in coronary heart disease patients. Circulation 126(12):1436-40.

Rosano C, Aizenstein H, Brach J, Longenberger A, Studenski S, Newman AB. 2008. Gait Measures Indicate Underlying Focal Gray Matter Atrophy in the Brain of Older Adults. J Gerontol A Biol Sci Med Sci 63(12):1380-1388.

Roubenoff R. 2000. Sarcopenic obesity: does muscle loss cause fat gain? Lessons from rheumatoid arthritis and osteoarthritis. Annals of the New York Academy of Sciences 904:553-7.

Salacinski AJ, Krohn K, Lewis SF, Holland ML, Ireland K, Marchetti G. 2012. The effects of group cycling on gait and pain-related disability in individuals with mild-to-moderate knee osteoarthritis: a randomized controlled trial. J Orthop Sports Phys Ther 42(12):985-95.

Sandstad J, Stensvold D, Hoff M, Nes BM, Arbo I, Bye A. 2015. The effects of high intensity interval training in women with rheumatic disease: a pilot study. European Journal of Applied Physiology 115(10):2081-9.

Senior HE, Henwood TR, Beller EM, Mitchell GK, Keogh JWL. 2015. Prevalence and risk factors of sarcopenia among adults living in nursing homes. Maturitas 82(4):418-423

Shiraev T, Barclay G. 2012. Evidence based exercise Clinical benefits of high intensity interval training. Australian Family Physician 41:960-962.

Tanaka R, Ozawa J, Kito N, Moriyama H. 2015. Does exercise therapy improve the health-related quality of life of people with knee osteoarthritis? A systematic review and meta-analysis of randomized controlled trials. Journal of Physical Therapy Science 27(10):3309-3314.

Trehan SK, Wolff AL, Gibbons M, Hillstrom HJ, Daluiski A. 2015. The effect of simulated elbow contracture on temporal and distance gait parameters. Gait Posture 41(3):791-4.

Tubach F, Ravaud P, Baron G, Falissard B, Logeart I, Bellamy N, Bombardier C, Felson D, Hochberg M, van der Heijde D, Dougados M. 2005. Evaluation of clinically relevant changes in patient reported 
outcomes in knee and hip osteoarthritis: the minimal clinically important improvement. Annals of the Rheumatic Diseases 64(1):29.

578 Uthman OA, van der Windt DA, Jordan JL, Dziedzic KS, Healey EL, Peat GM, Foster NE. 2013. Exercise for

579

580

581

582

583

584

585

586

587

588 lower limb osteoarthritis: systematic review incorporating trial sequential analysis and network meta-analysis. BMJ 347:f5555.

Wellsandt E, Golightly Y. 2018. Exercise in the management of knee and hip osteoarthritis. Curr Opin Rheumatol 30(2):151-159.

Yoshimura N, Muraki S, Oka H, Tanaka S, Kawaguchi H, Nakamura K, Akune T. 2012. Accumulation of metabolic risk factors such as overweight, hypertension, dyslipidaemia, and impaired glucose tolerance raises the risk of occurrence and progression of knee osteoarthritis: a 3-year follow-up of the ROAD study. Osteoarthritis Cartilage 20(11):1217-26. 
Figure 1 (on next page)

Figure 1: Participant CONSORT flow diagram 
Figure 1: Participant CONSORT flow diagram.

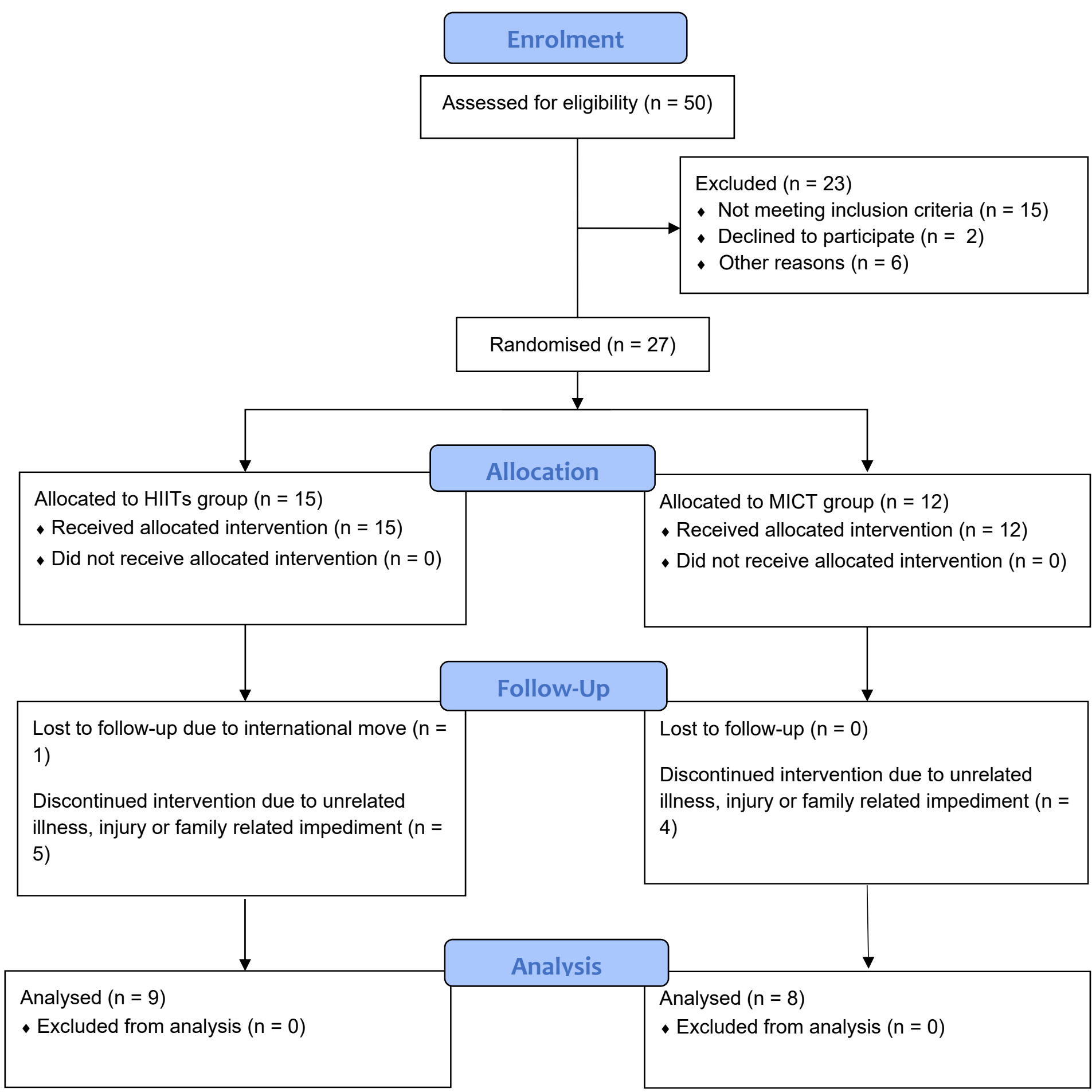




\section{Table $\mathbf{1}$ (on next page)}

Table 1: Baseline characteristics of the participants who completed the trial.

All results are mean (standard deviation) except where indicated. HIIT = high intensity interval training cycling, $\mathrm{MICT}=$ moderate intensity continuous cycling, $\mathrm{M}=$ male, $\mathrm{F}=$ female, $L=$ left, $R=$ right. 
1 Table 1: Baseline characteristics of the participants who completed the trial.

\begin{tabular}{lcccc}
\hline & & & \multicolumn{3}{c}{ Between } & \\
& HIIT $(\mathrm{n}=9)$ & MICT $(\mathrm{n}=8)$ & group p-value & All (n=17) \\
\hline Age (years) & $59.1(6.7)$ & $66.1(8.8)$ & 0.09 & $62.4(8.3)$ \\
Gender (M / F) & $3 / 6$ & $1 / 7$ & 0.31 & $4 / 13$ \\
Height (cm) & $170.0(6.2)$ & $165.5(6.3)$ & 0.16 & $167.9(6.5)$ \\
Body mass (kg) & $78.5(13.5)$ & $77.8(23.0)$ & 0.94 & $78.2(18.0)$ \\
BMI & $27.0(4.0)$ & $28.2(6.9)$ & 0.69 & $27.6(5.4)$ \\
Osteoarthritis side (Both / L / R) & $4 / 3 / 2$ & $4 / 0 / 4$ & 0.67 & $8 / 3 / 6$ \\
Duration of diagnosis (years) & $4.6(5.8)$ & $4.9(3.2)$ & 0.91 & $4.7(4.6)$ \\
Prior Surgery & 5 & 6 & 0.40 & 11 \\
WOMAC & $36.1(15.0)$ & $34.8(15.5)$ & 0.85 & $35.5(14.8)$ \\
Lequesne index & $8.8(4.3)$ & $9.6(3.9)$ & 0.69 & $9.0(4.2)$ \\
\hline All results a & & & & \\
\hline
\end{tabular}

2 All results are mean (standard deviation) except where indicated. HIIT = high intensity interval training

3 cycling, $\mathrm{MICT}=$ moderate intensity continuous cycling, $\mathrm{M}=$ male, $\mathrm{F}=$ female $\mathrm{L}=$ left, $\mathrm{R}=$ right. 


\section{Table 2 (on next page)}

Table 2: Exercise adherence and adverse events.

All results are mean (standard deviation) except where indicated. HIIT = high intensity interval training cycling, MICT = moderate intensity continuous cycling. 
1 Table 2: Exercise adherence and adverse events.

\begin{tabular}{lccc}
\hline & HIIT (n=9) & MICT (n=8) & All (n= 17) \\
\hline Adherence (\%) & $94(8)$ & $88(12)$ & $91(10)$ \\
Adverse events (number) & 26 & 2 & 28 \\
Participants reporting adverse events (number) & 2 & 1 & 3
\end{tabular}

$2 \overline{\text { All results are mean (standard deviation) except where indicated. HIIT = high intensity interval training }}$

3 cycling, $\mathrm{MICT}=$ moderate intensity continuous cycling. 


\section{Table 3(on next page)}

Table 3: Training-related changes in health-related quality of life, functional performance and body composition.

All results are mean (standard deviation). HIIT = high intensity interval training cycling, MICT

$=$ moderate intensity continuous cycling. ${ }^{*}=$ significant pre-post test (within-group) effect, $\uparrow$ = significant between-group effect favouring the HIIT group. 
1 Table 3: Training-related changes in health-related quality of life, functional performance and body composition.

\begin{tabular}{|c|c|c|c|c|c|c|c|c|c|}
\hline \multirow{2}{*}{ 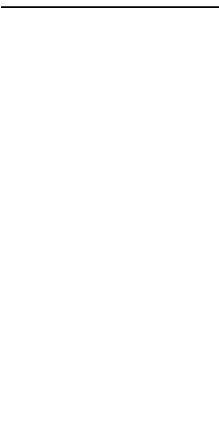 } & \multicolumn{4}{|c|}{ HIIT $(n=9)$} & \multicolumn{4}{|c|}{$\mathrm{MICT}(\mathrm{n}=8)$} & \multirow[b]{2}{*}{$\begin{array}{l}\text { Betwee } \\
\text { n-Group } \\
\text { P Value }\end{array}$} \\
\hline & Pre-test & Post-test & $\begin{array}{l}\text { Pre-post } \\
\text { HIIT } \\
\text { P Value }\end{array}$ & $\begin{array}{l}\text { Pre-post HIIT } \\
\text { effect size } \\
(95 \% \mathrm{Cl})\end{array}$ & Pre-test & Post-test & $\begin{array}{l}\text { Pre- } \\
\text { post } \\
\text { MICT } \\
\text { P Value }\end{array}$ & $\begin{array}{l}\text { Pre-post MICT } \\
\text { effect size } \\
\qquad(95 \% \mathrm{Cl})\end{array}$ & \\
\hline WOMAC & $36.1(15.0)$ & $\begin{array}{c}21.2(14.6) \\
*\end{array}$ & 0.005 & $0.91(0.28-1.70)$ & $\begin{array}{l}34.8 \\
(15.5)\end{array}$ & $\begin{array}{c}22.9(14.4) \\
*\end{array}$ & 0.006 & $0.71(0.21-1.36)$ & 0.829 \\
\hline $\begin{array}{l}\text { Lequesne } \\
\text { index }\end{array}$ & $8.8(4.3)$ & 6.7 (3.9) & 0.102 & $0.46(-0.10-1.10)$ & $9.6(3.9)$ & $8.4(4.6)$ & 0.081 & $\begin{array}{c}0.25(-0.04- \\
0.59)\end{array}$ & 0.707 \\
\hline TUG (s) & $8.9(2.0)$ & $7.8(1.1)^{*}+$ & 0.004 & $0.62(0.14-1.20)$ & $9.1(1.9)$ & $9.7(3.5)$ & 0.401 & $\begin{array}{c}-0.19(-0.69- \\
0.28)\end{array}$ & 0.043 \\
\hline $\begin{array}{l}\text { Sit to Stand } \\
\text { (reps) }\end{array}$ & $11.1(2.2)$ & $13.1(2.7)$ * & 0.012 & $0.73(0.17-1.43)$ & 9.4 (1.9) & $10.6(3.5)$ & 0.095 & $\begin{array}{c}0.38(-0.10- \\
0.92)\end{array}$ & 0.417 \\
\hline $\begin{array}{l}\text { Gait speed } \\
(\mathrm{m} / \mathrm{s})\end{array}$ & $1.21(0.18)$ & $1.21(0.14)$ & 0.883 & $0.00(-0.54-0.54)$ & $\begin{array}{c}1.11 \\
(0.17)\end{array}$ & $1.14(0.20)$ & 0.706 & $\begin{array}{c}0.14(-0.68- \\
0.99)\end{array}$ & 0.685 \\
\hline Body mass & 78.5 (13.5) & $78.7(13.6)$ & 0.605 & $-0.01(-0.02-$ & 77.8 & $77.8(22.1)$ & 0.978 & $0.00(-0.03-$ & 0.715 \\
\hline
\end{tabular}




\begin{tabular}{|c|c|c|c|c|c|c|c|c|c|}
\hline (kg) & & & & $0.00)$ & (23.0) & & & $0.03)$ & \\
\hline BMI & $27.0(4.0)$ & 26.6 (3.9) & 0.426 & $0.09(0.05-0.15)$ & $28.2(7.3)$ & $28.2(6.9)$ & 0.810 & $0.00(-0.04-$ & 0.784 \\
\hline$\left(\mathrm{kg} / \mathrm{m}^{2}\right)$ & & & & & & & & $0.04)$ & \\
\hline Body fat & $30.9(5.6)$ & $31.4(5.7)$ & 0.197 & $-0.11(-0.93-$ & $36.0(9.0)$ & $36.6(9.1)$ & 0.099 & $-0.05(-0.17-$ & 0.967 \\
\hline (\%) & & & & 0.69) & & & & $0.04)$ & \\
\hline Muscle & $51.6(10.7)$ & $51.3(10.5)$ & 0.179 & $-0.02(-0.07-0.12)$ & $45.9(7.4)$ & $45.5(7.2)$ & 0.136 & $-0.05(-0.16-$ & 0.861 \\
\hline mass (kg) & & & & & & & & $0.05)$ & \\
\hline
\end{tabular}

2 All results are mean (standard deviation). HIIT = high intensity interval training cycling, MICT = moderate intensity continuous cycling.

$3^{*}=$ significant pre-post test (within-group) effect, $\uparrow=$ significant between-group effect favouring the HIIT group. 